\title{
Reactivity of Porphyrazines in Acid-Base Interaction with $N$-Bases
}

\author{
Oleg A. Petrov, ${ }^{@}$ Galina V. Osipova, and Olga G. Khelevina
}

Ivanovo State University of Chemistry and Technology, Ivanovo, 153000, Russia

@Corresponding authorE-mail: poa@isuct.ru

\begin{abstract}
The analysis of porphyrazine reactivity in the processes of acid-base interaction with proton acceptor molecules, containing nitrogen atom, in benzene solutions is carried out. It is shown that tetrahalogenoporphyrazines are evident as dibasic NH-acid in relation to organic bases such as pyridine, 2-methylpyridine, morpholine, benzylamine, n-butylamine, tert-butylamine, tri-n-butylamine, diethylamine, triethylamine or piperidine and form H-complexes with proton transfer, which are subjected to the kinetic process of destruction. In contrast, substituted phthalocyanines in system benzene (DMSO) - morpholine (benzylamine) form kinetically stable complexes with proton transfer. It is found that intermolecular transfer of $\mathrm{NH}$-group protons from porphyrazine macrocycle to the organic base is a remarkably slow process $\left(k^{298}=10^{-2}-10^{-6} \mathrm{l} \cdot \mathrm{s}^{-n} \cdot \mathrm{mol}^{-n}\right)$ and is described by the total kinetic equation of the second or third order. The correlation dependence for tetrahalogenoporphyrazines between the reaction rate and $p K_{a}$ value of nitrogen containing bases is revealed. It is found that spatial screening of proton-acceptor centre prevents a favorable contact of acidbase centers of interacting molecules and obstructs the proton transfer from the acid to the base. It is shown that modification of porphyrin macrocycle owing to tetraazasubstitution and benzoannulation effects on the acid properties of the molecule. When passing from tetrahalogenoporphyrazines to substituted phthalocyanines the rate of the proton transfer decreases by several orders of magnitude on the background of growing $E_{a}$ and $\triangle S$ characteristics. When changing benzene for DMSO the proton drawing from the macrocyclic plane is facilitated under action of the base.
\end{abstract}

Keywords: Porphyrazines, organic bases, acid-base interaction.

Петров Олег Александрович, родился в 1966 г. в г. Тейково, Ивановской области. В 1988-1991 г2. обучался в очной аспирантуре ИХТИ под руководством Б.Д. Березина и О.Г. Хелевиной. В 1992 г. защитил кандидатскую диссертацию на тему "Влияние природы растворителя на кинетику образования комплексов тетраазапорфиринов". С 1991 по 1995 гг. работал на кафедре органической химии ИХТИ, возглавляемой Б.Д. Березиным. В 2004 г. защитил докторскую диссертащию на тему "Реакиионная способность тетраазапорфиринов в прочессах кислотноосновного взаимодействия и образование молекулярных комплексов". С 1991 г. по настоящее время работает на кафедре органической химии ИГХТУ и занимается исследованиями физико-химических свойств тетразапорфиринов и их аналогов.

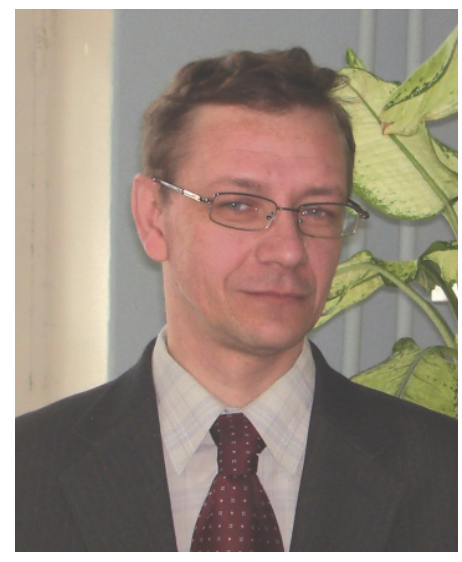

Professor Petrov Oleg Aleksandrovich was born in 1966 in Teikovo, Ivanovo Region. In 1988-1991 he was post-graduate student at Ivanovo Institute of Chemical Technology under the supervision of Prof. B.D. Berezin and Prof. $O . G$. Khelevina and in 1992 received the Degree "Candidate of Chemical Sciences" (PhD) presenting the dissertation "Influence of the solvent nature on kinetics of tetraazaporphyrin complexation". In 1991-1995 he held teaching positions in the Department of Organic Chemistry at Ivanovo University of Chemical Technology (ISUCT) headed by Prof. B.D. Berezin. In 2004 he received the degree "Doctor of Chemical Sciences" for the dissertation "Reactivity of tetraazaporphyrins in the acid-base interaction processes and formation of molecular complexes". Currently he has professor position in the Department of Organic Chemistry (ISUCT) and his research interests are connected with physical chemical properties of tetraazaporphyrins and their analogues.
Осипова Галина Вячеславовна, родилась в 1979 г. вг. Иваново. В 2002-2005 г2. обучалась в очной аспирантуре ИГХТУ под руководством Б.Д. Березина и О.А. Петрова. В 2005 г. защитила кандидатскую диссертацию на тему: "Реакиионная способность $\beta$-замещеенных и $\beta, \beta$-аннелированных порфиразинов в процессах кислотно-основного взаимодействия". С 2006 года по настоящее время работает на кафедре химии и технологии высокомолекулярных соединений ИГХТУ и занимается иммобилизачией металлокомплексов фталочианинов на химически модифицированные полимеры.

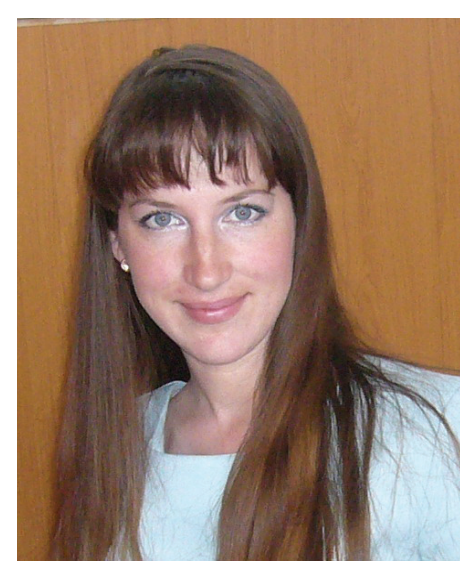

Dr. Osipova Galina Vyacheslavovna was born in 1979 in Ivanovo. In 2002-2005 she was post-graduate student at Ivanovo State University of Chemical Technology (ISUCT) under the supervision of Prof. B.D. Berezin and Dr. O.A. Petrov and in 2005 received the Degree "Candidate of Chemical Sciences" (PhD) presenting the dissertation "Reactivity of $\beta$-substituted and $\beta, \beta$-annulated porphyrazines in acid-base interaction processes". Currently she has a research position in the Department of High Molecular Weight Compounds at ISUCT and studies the immobilization of metal phthalocyanines on chemically modified polymers. 


\title{
Реакционная способность порфиразинов в кислотно-основном взаимодействии с $\boldsymbol{N}$-основаниями
}

\author{
О.А. Петров, ${ }^{@}$ Г.В. Осипова, О.Г. Хелевина
}

Ивановский государственный химико-технологический университет, Иваново, 153000, Россия

@E-mail:poa@isuct.ru

\begin{abstract}
В обзоре проведен анализ реакиионной способности порфиразинов в кислотно-основном взаимодействии с азотсодержсащими протоно-акиепторныли молекулами в бензоле. Установлены необычно низкие скорости проиесса. Показано влияние природы заместителя в пиррольных кольиах макроцикла на кислотные свойства молекульл. Обсуждена взаимосвязь между электронным и геометрическим строением основания и кинетическими параметрами межмолекулярного переноса протонов NH-групп порфиразинов.
\end{abstract}

Ключевые слова: Порфиразины, органические основания, кислотно-основное взаимодействие.

Реакции с участием кислот и оснований представляют собой обширный и важный раздел современной химии. Они широко используются в синтезе и технологии, а также играют ключевую роль в ряде жизненно важных процессов. В связи с этим изучение реакций кислотно-основного взаимодействия (КОВ) с участием тетрапиррольных макроциклов представляет несомненный интерес, поскольку они входят в состав многих биохимических систем. В качестве модельных соединений для исследования КОВ первоначально могут быть использованы порфиразины, являющиеся структурными аналогами биопорфиринов.

Порфиразины обладают выраженной кислотностью по внутрициклическим NH-связям, ${ }^{[1,2]}$ которая объясняет факт их взаимодействия с основаниями $(B)$. Количественные данные об этих реакциях весьма немногочисленны. Наиболее полная информация получена лишь для тетрагалогенпорфиразинов $\left(\mathrm{H}_{2} \mathrm{PAR}_{4}\right) \cdot{ }^{[3-7]}$

Согласно, ${ }^{[3,4,6]}$ тетрагалогенпорфиразины в присутствии азотсодержащих оснований проявляют свойства двухосновных NH-кислот и образуют с течением времени лабильные комплексы с переносом протонов $\mathrm{H}_{2} \mathrm{PAR}_{4} \cdot 2 B$.

Детальное изучение кислотно-основного взаимодействия с участием $\mathrm{H}_{2} \mathrm{PAR}_{4}$ показало, что в системе бензол- $B$ реакция

$$
\mathrm{H}_{2} \mathrm{PAR}_{4}+2 \mathrm{~B} \rightarrow \mathrm{H}_{2} \mathrm{PAR}_{4} \cdot 2 \mathrm{~B}
$$

описывается кинетическим уравнением:

$$
-\mathrm{d} C_{\mathrm{H}_{2} \mathrm{PAR}_{4}} / \mathrm{d} \tau=k C_{\mathrm{H}_{2} \mathrm{PAR}_{4}} C^{n}{ }_{B},
$$

где $B$ - пиридин (Ру), 2-метилпиридин (МеРу), морфолин (Mor), триэтиламин $\left(\mathrm{Et}_{3} \mathrm{~N}\right)$, три-н-бутиламин $\left(\mathrm{Bu}_{3} \mathrm{~N}\right)$, пиперидин (Рip), бензиламин $\left(\mathrm{BzNH}_{2}\right), \quad$-бутиламин $\left(\mathrm{BuNH}_{2}\right)$, mрет-бутиламин $\left(\mathrm{Bu}^{\mathrm{t}} \mathrm{NH}_{2}\right)$ и диэтиламин $\left(\mathrm{Et}_{2} \mathrm{NH}\right)$, а $n$ - порядок реакции по основанию.

Оказалось, что при взаимодействии $\mathrm{H}_{2} \mathrm{PAR}_{4}$ c циклическими и третичными ациклическими аминами значение $n$ близко к единице. Лимитирующая стадия процесса в этом случае имеет бимолекулярный характер, а перенос протонов $\mathrm{NH}-г р у п п ~ \mathrm{H}_{2} \mathrm{PAR}_{4}$ осуществляется двухстадийно. ${ }^{[3,6]}$ Для первичных и вторичных ациклических аминов порядок реакции по $B$ близок к 2. Очевидно, что в этом случае лимитирующей стадией является не тримолекулярный процесс, а бимолекулярная реакция между $\mathrm{H}_{2} \mathrm{PAR}_{4}$ и Н-связанными димерными молекулами оснований. При этом не исключается возможность протекания процесса в две стадии с соизмеримыми значениями констант скоростей $k_{1}$ и $k_{2} \cdot{ }^{[6]}$

Важно отметить, что межмолекулярный перенос протонов NH-групп от $\mathrm{H}_{2} \mathrm{PAR}_{4}$ к $B$ характеризуется низкими скоростями (Таблица 1), обусловленными действием макроциклического эффекта, включающего в себя как стерическую, так и электронную составляющую. ${ }^{[8,9]}$ Последняя характеризует изменение степени ковалентности связей NH в основном за<smiles>[R][R]=[R]=[R]=[R]=[R]=[R]</smiles>

$\left(\mathrm{H}_{2} \mathrm{PABr}_{4}\right.$ - тетрабромтетраазапорфин);

$\left(\mathrm{H}_{2} \mathrm{PACl}_{4}\right.$ - тетрахлортетраазапорфин). 
Таблица 1. Кинетические параметры реакции кислотно-основного взаимодействия $\mathrm{H}_{2} \mathrm{PAR}_{4}$ с основаниями в бензоле $\left(C_{\mathrm{H}_{2} \mathrm{PAR}_{4}}^{\mathrm{o}}=(0,4-0,5) \cdot 10^{-5}\right.$ моль/л $) \cdot{ }^{[3,4]}$

\begin{tabular}{|c|c|c|c|c|}
\hline $\mathrm{H}_{2} \mathrm{PAR}_{4}$ & Основание & $k^{298} \cdot 10^{4}, \pi^{\mathrm{n}} /\left(\right.$ моль $\left.\mathrm{b}^{\mathrm{n}} / \mathrm{c}\right)$ & $E_{\mathrm{a}}$, кДж/моль & $\Delta S^{\ddagger}$, Дж/(моль·К) \\
\hline \multirow[t]{10}{*}{$\mathrm{H}_{2} \mathrm{PABr}_{4}$} & Пиридин & 0,23 & 69 & -92 \\
\hline & 2-Метилпиридин & 0,015 & 87 & -54 \\
\hline & Морфолин & $0,72^{*}$ & 26 & -224 \\
\hline & Триэтиламин & 0,24 & 46 & -165 \\
\hline & Три-н-бутиламин & 0,086 & 26 & -262 \\
\hline & Пиперидин & $28,3^{*}$ & 20 & -237 \\
\hline & Бензиламин & $0,78^{*}$ & 29 & -226 \\
\hline & н-Бутиламин & $6,6^{*}$ & 11 & -270 \\
\hline & mpem- Бутиламин & $0,076^{*}$ & 18 & -283 \\
\hline & Диэтиламин & $4,0^{*}$ & 15 & -261 \\
\hline \multirow[t]{10}{*}{$\mathrm{H}_{2} \mathrm{PACl}_{4}$} & Пиридин & 0,36 & 40 & -188 \\
\hline & 2-Метилпиридин & 0,04 & 49 & -174 \\
\hline & Морфолин & $0,80^{*}$ & 28 & -220 \\
\hline & Триэтиламин & 1,10 & 30 & -214 \\
\hline & Три-н-бутиламин & 0,23 & 24 & -260 \\
\hline & Пиперидин & $22,8^{*}$ & 23 & -229 \\
\hline & Бензиламин & $0,65^{*}$ & 31 & -223 \\
\hline & н-Бутиламин & $3,8^{*}$ & 23 & -235 \\
\hline & mpem- Бутиламин & $0,09^{*}$ & 28 & -251 \\
\hline & Диэтиламин & $1,10^{*}$ & 30 & -218 \\
\hline
\end{tabular}

$*-k^{298} \cdot 10^{2}$

счет передачи электронных эффектов заместителей с периферии молекулы на реакционный центр $\mathrm{H}_{2} \mathrm{~N}_{4}$. При переходе от порфиринов к порфиразинам на фоне изменения энергетической разности между граничными $\pi$-молекулярными орбиталями (ВЗМО и НСМО) $[1,10]$ происходит увеличение полярности связей $\mathrm{NH}$ и, как следствие, уменьшение наиболее энергоемкой составляющей, связанной с растяжением связей в ходе формирования переходного состояния. В результате этого создаются благоприятные условия для переноса протонов групп $\mathrm{NH}$ от $\mathrm{H}_{2} \mathrm{PAR}_{4}$ к $B$. С другой стороны, рост ароматичности и конформационной жесткости макроцикла при тетраазазамещении вызывает в $\mathrm{H}_{2} \mathrm{PAR}_{4}$ одновременное увеличение стерической составляющей, которая характеризует экранирование атомами и $\pi$-электронами протонов NH-групп. Эта составляющая действует в противовес электронной компоненте и, повидимому, вносит основной вклад в кинетику процесса, препятствуя оптимальной пространственной ориентации молекул-партнеров в ходе КОВ и затрудняя наиболее благоприятный контакт их реакционных центров.

Из данных Таблицы 1 видно, что электронные эффекты атомов галогенов, передающиеся на реакционный центр макроцикла с полуизолированных $\mathrm{C}_{\beta}=\mathrm{C}_{\beta}$ связей, не оказывают существенного влияния на кислотно-основное взаимодействие, а отрыв протонов от их NН-групп под влиянием $B$ осуществляется в $\sim 10^{8}$ $10^{11}$ раз труднее, чем от донорных молекул, имеющих открытые, т.е. пространственно не экранированные кислотные центры, блокированные сильной внутримолекулярной Н-связью. ${ }^{[1,12]}$
Дальнейшие исследования показали, что скорость и активационные параметры КОВ достаточно сильно зависят от природы протоноакцептора. Обнаружено, ${ }^{[3,7]}$ что с увеличением $\mathrm{p} K$ азотсодержащих оснований скорость межмолекулярного переноса протонов $\mathrm{NH}$-групп $\mathrm{H}_{2} \mathrm{PABr}_{4}$ и $\mathrm{H}_{2} \mathrm{PACl}_{4}$ возрастает, а энергия активации процесса уменьшается (Таблица 1). При этом выполняется линейное соотношение между $\lg k^{298}$ и $\mathrm{p} K_{\mathrm{a}}$ (Рисунок 1) и наблюдается симбатное изменение величин $E_{\text {а и }} \Delta \mathrm{S}^{\ddagger}$, свидетельствующее о наличии кинетического компенсационного эффекта (Рисунок 2). Среди всех изученных циклических оснований максимальной реакционной способностью при взаимодействии с $\mathrm{H}_{2} \mathrm{PAR}_{4}$ обладает пиперидин, который имеет стерически доступный атом азота в составе молекулы ${ }^{[13]}$ и является достаточно сильным акцептором протона $\left(\mathrm{p} K_{\mathrm{a}}=11,23^{[14]}\right)$. Введение в молекулу пиперидина атома кислорода не влияет на пространственное строение основания, ${ }^{[13]}$ однако приводит к снижению $K_{\mathrm{a}}$ на $\sim 2,5$ порядка и, как следствие, к уменьшению скорости КОВ. При переходе к пиридину $\left(\mathrm{p} K_{\mathrm{a}}=5,23^{[14]}\right)$ наблюдается дальнейшее ингибирование процесса, что, прежде всего, объясняется низкой протоно-акцепторной способностью этого основания по сравнению с пиперидином и морфолином. Минимальные скорости и максимальный энергетический барьер имеют место при взаимодействии $\mathrm{H}_{2} \mathrm{PAR}_{4} \mathrm{c}$ 2-метилпиридином $\left(\mathrm{p} K_{\mathrm{a}}=5,97^{[14]}\right)$, который полностью выпадает из корреляционной зависимости $\lg k^{298}=f\left(\mathrm{p} K_{\mathrm{a}}\right)$ (Рисунок 1), вследствие более сильного, чем в пиридине, пространственного экранирования неподеленной электронной пары атома азота. Аналогичный вклад 


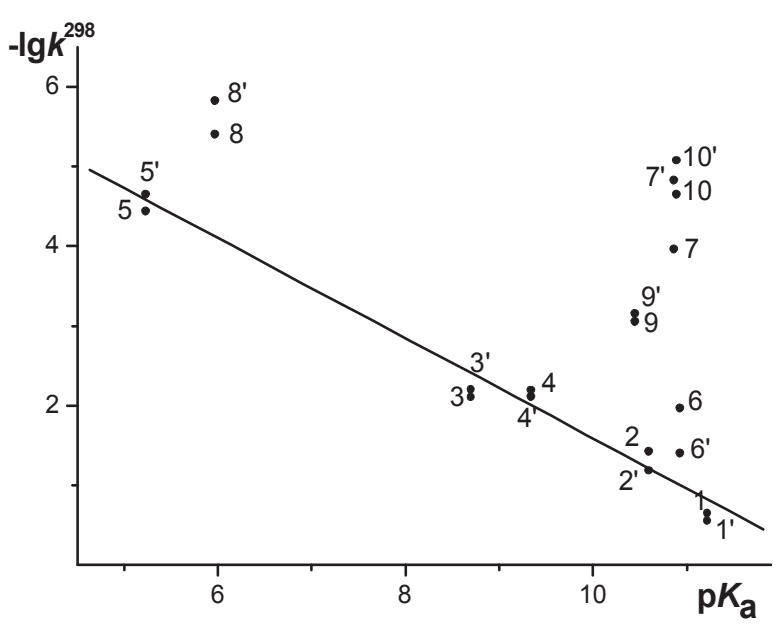

Рисунок 1. Зависимость $\lg k^{298}$ от $\mathrm{p} K_{\mathrm{a}}$ для реакций $\mathrm{H}_{2} \mathrm{PACl}_{4}(1-$ 10) и $\mathrm{H}_{2} \mathrm{PABr}_{4}\left(1^{\prime}-10^{\prime}\right)$ с пиперидином $\left(1,1^{\prime}\right), \boldsymbol{\mu}$-бутиламином $\left(2,2^{\prime}\right)$, морфолином $\left(3,3^{\prime}\right)$, бензиламином $\left(4,4^{\prime}\right)$, пиридином $\left(5,5^{\prime}\right)$, диэтиламином $\left(6,6^{\prime}\right)$, триэтиламином $\left(7,7^{\prime}\right)$, 2-метилпиридином $\left(8,8^{\prime}\right)$, трет-бутиламином $\left(9,9^{\prime}\right)$, трибутиламином $\left(10,10\right.$ ') в бензоле при Т=298 К. $\left(-\lg k^{298}=7,86-\right.$ $0,62 \cdot \mathrm{p} K$, коэффициент линейной корреляции $-0,98427)$

пространственного фактора в реакцию переноса протона от NH-кислоты к основанию наблюдается при замене $\mathrm{BuNH}_{2}\left(\mathrm{p} K_{\mathrm{a}}=10,60^{[14]}\right)$ наEt $_{2} \mathrm{NH}\left(\mathrm{p} K_{\mathrm{a}}=10,93^{[14]}\right)$, а также $\mathrm{Et}_{3} \mathrm{~N}$ $\left(\mathrm{p} K_{\mathrm{a}}=10,87^{[14]}\right)$ на $\mathrm{Bu}_{3} \mathrm{~N}\left(\mathrm{p} K_{\mathrm{a}}=10,89^{[14]}\right)$ (Таблица 1$)$. Наряду с увеличением числа и длины алкильных заместителей оптимальной пространственной ориентации кислотноосновных центров противодействует разветвление углеводородной цепи в амине. Так, скорости переноса протонов $\mathrm{NH}-г р у п п ~ к ~ \mathrm{BuNH}_{2}$ и $\mathrm{Bu}^{\mathrm{t}} \mathrm{NH}_{2}\left(\mathrm{p} K_{\mathrm{a}}=10,45^{[14]}\right)$ различаются в 90 и 40 раз для $\mathrm{H}_{2} \mathrm{PABr}_{4}$ и $\mathrm{H}_{2} \mathrm{PACl}_{4}$ соответственно (Таблица 1). Подобное ингибирование<smiles>CCCCCCCCCCCC(C)(C)C</smiles>

$\left(\mathrm{H}_{2} \mathrm{Pc}\left(\mathrm{Bu}^{\mathrm{t}}\right)_{4}\right)$

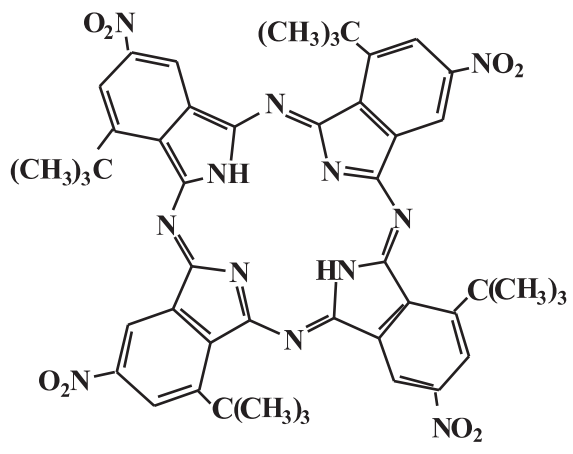

$\left(\mathrm{H}_{2} \mathrm{Pc}\left(\mathrm{NO}_{2}\right)_{4}\left(\mathrm{Bu}^{\mathrm{t}}\right)_{4}\right)$

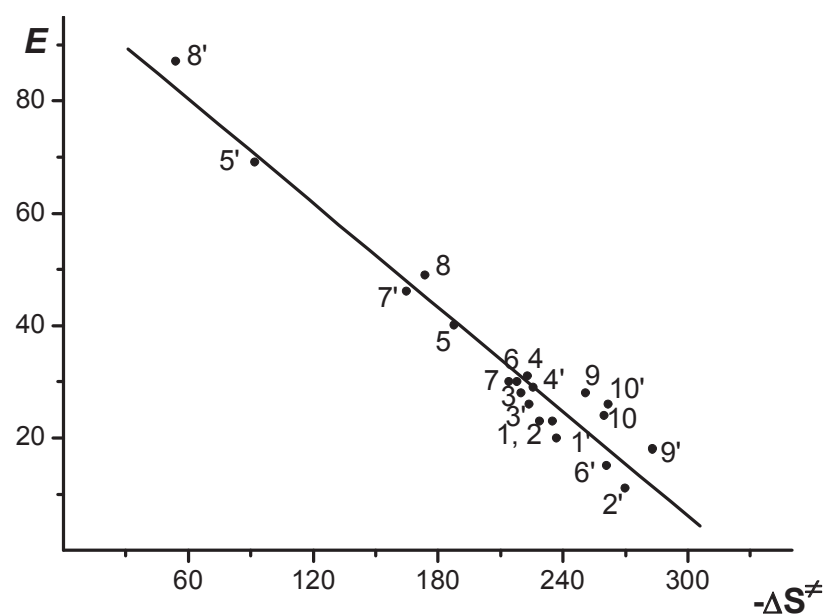

Рисунок 2. Кинетический компенсационный эффект реакции $\mathrm{H}_{2} \mathrm{PACl}_{4}$ (1-10) и $\mathrm{H}_{2} \mathrm{PABr}_{4}\left(1^{\prime}-10\right.$ ') с пиперидином(1, 1'), $н$-бутиламином (2, 2'), морфолином (3, 3'), бензиламином $\left(4,4^{\prime}\right)$, пиридином $\left(5,5^{\prime}\right)$, диэтиламином $\left(6,6^{\prime}\right)$, триэтиламином (7, 7'), 2-метилпиридином (8, 8'), mрет-бутиламином $\left(9,9^{\prime}\right)$, трибутиламином $\left(10,10^{\prime}\right)$ в бензоле. $\left(E=98,94+0,31 \cdot \Delta \mathrm{S}^{\neq}\right.$, коэффициент линейной корреляции $-0,97133)$

процесса межмолекулярного переноса протона ранее отмечалось для относительно простых кислотноосновных систем. Скорости таких реакций при переходе от вторичного амина к третичному уменьшались в 2-6 раз, оставаясь при этом сравнительно высокими $\left(k=10^{4}-10^{8}\right.$ л/(моль·с)). ${ }^{[1,12]}$

Известно, ${ }^{[15]}$ что модификация порфиринового макроцикла за счет тетраазазамещения и бензоаннелирования, приводящего к расширению $\pi$-системы хромофора, оказывает существенноевлияние на электронное и геометрическое строение молекулы и, как следствие, на ее кислотные свойства. Для выявления влияния такой структурной модификации были взяты тетра(3-нитро-5-трет-бутил)фталоцианин $\left.\mathrm{H}_{2} \mathrm{Pc}\left(\mathrm{NO}_{2}\right)_{4}{ }^{(} \mathrm{Bu}\right)_{4} \quad$ и тетра(4-mpem-бутил)фталоцианин $\mathrm{H}_{2} \mathrm{Pc}\left(\mathrm{Bu}^{\mathrm{t}}\right)_{4}$.

Исследование состояния тетра(3-нитро-5-mpemбутил)фталоцианина в системе бензол-Ру (МеРу, Mor, $\left.\mathrm{BzNH}_{2}\right)$ показало, что $\mathrm{H}_{2} \mathrm{Pc}\left(\mathrm{NO}_{2}\right)_{4}\left({ }^{\mathrm{t}} \mathrm{Bu}\right)_{4}$ вступает в кислотно-основное взаимодействие только с морфолином и бензиламином. При этом он ведет себя как двухосновная NH-кислота, образуя кинетически устойчивые комплексы с переносом протонов $\mathrm{H}_{2} \mathrm{Pc}\left(\mathrm{NO}_{2}\right)_{4}\left({ }^{\mathrm{t}} \mathrm{Bu}\right)_{4} \cdot 2 \mathrm{~B}\left(\mathrm{~B}: \mathrm{BzNH}_{2}, \mathrm{Mor}\right) \cdot{ }^{[16]}$

Изучение специфики образования этих комплексов показало, что реакция

$$
\mathrm{H}_{2} \mathrm{Pc}\left(\mathrm{NO}_{2}\right)_{4}{ }^{\left({ }^{\mathrm{B}} \mathrm{Bu}\right)_{4}}+2 \mathrm{~B} \rightarrow \mathrm{H}_{2} \mathrm{Pc}\left(\mathrm{NO}_{2}\right)_{4}\left({ }^{\mathrm{t}} \mathrm{Bu}\right)_{4} \cdot 2 B
$$

описывается кинетическим уравнением второго порядка:

$$
-\mathrm{d} C_{\mathrm{H}_{2} \mathrm{Pc}\left(\mathrm{NO}_{2}\right)_{4}\left({ }^{\mathrm{t} B u}\right)_{4}} / \mathrm{d} \tau=k \cdot C_{\mathrm{H}_{2} \mathrm{Pc}\left(\mathrm{NO}_{2}\right)_{4}{ }^{\mathrm{t}}{ }^{\mathrm{Bu})_{4}}} \cdot C_{B}
$$

и в отличие от реакции (1) характеризуется более низкими скоростями отрыва протонов NН-групп (Таблицы 1, 2). 
Таблица 2. Кинетические параметры реакции кислотноосновного взаимодействия $\mathrm{H}_{2} \mathrm{Pc}\left(\mathrm{NO}_{2}\right)_{4}\left(\mathrm{Bu}^{\mathrm{t}}\right)_{4}$ с основаниями в бензоле $\left(\mathrm{C}_{\left.\mathrm{H}_{2} \mathrm{Pc}^{\circ} \mathrm{NO}_{2}\right)_{4}{ }_{\left(\mathrm{Bu}^{\mathrm{t}}\right)_{4}}^{\mathrm{t}}}=0,9 \cdot 10^{-5}\right.$ моль/л $\left.{ }^{[16]}\right)$.

\begin{tabular}{lccc}
\hline Основание & $\begin{array}{c}k^{298} \cdot 10^{4}, \\
\pi /(\text { моль } / \mathrm{c})\end{array}$ & $\begin{array}{c}E_{\mathrm{a}}, \\
\text { кДж/моль }\end{array}$ & $\begin{array}{c}\Delta \mathrm{S}^{ \pm}, \\
\text {Дж/(моль К) }\end{array}$ \\
\hline Морфолин & 0,025 & 45 & -356 \\
Бензиламин & 0,63 & 32 & -338 \\
\hline
\end{tabular}

По реакционной способности тетра(3-нитро-5трет-бутил)фталоцианин занимает промежуточное положение между $\mathrm{H}_{2} \mathrm{PR}_{4}$ и $\mathrm{H}_{2} \mathrm{Pc}\left({ }^{\mathrm{t}} \mathrm{Bu}\right)_{4}$. В отличие от тетрагалогенпорфиразинов он оказывается неактивным при взаимодействии с пиридином и 2-метилпиридином в бензольном растворе. ${ }^{[16]}$ В реакции с морфолином скорость кислотно-основного взаимодействия $\mathrm{H}_{2} \mathrm{Pc}\left(\mathrm{NO}_{2}\right)_{4}\left({ }^{\mathrm{t}} \mathrm{Bu}\right)_{4}$ и $\mathrm{H}_{2} \mathrm{PAR}_{4}$, судя по величинам $k^{298}$, различается на несколько порядков на фоне роста $E_{\text {а }}$ и $\Delta \mathrm{S}^{\neq}$процесса (Таблицы 1,2).

Несмотря на структурную близость замещенных фталоцианина, перенос протонов $\mathrm{NH}-г$ рупп $\mathrm{H}_{2} \mathrm{Pc}\left({ }^{\mathrm{t}} \mathrm{Bu}\right)_{4}$ в системе бензол-Ру (MePy, Mor, BzNH${ }_{2}$ ) не наблюдается. Кинетически контролируемое взаимодействие имеет место только в системе диметилсульфоксид-морфолин (бензиламин), обладающей достаточно высокой основностью, полярностью и сольватирующей способностью (Таблица 3). При этом реакция

$$
\mathrm{H}_{2} \mathrm{Pc}\left({ }^{\mathrm{t}} \mathrm{Bu}\right)_{4}+B \rightarrow \mathrm{H}_{2} \mathrm{Pc}\left({ }^{\mathrm{t}} \mathrm{Bu}\right)_{4} \cdot 2 B
$$

протекает в узком интервале концентраций морфолина и бензиламина и характеризуется низкими значениями констант скоростей и достаточно высокими значениями $E_{\text {a }}$ процесса. ${ }^{[17]}$ Напротив, в случае с пиперидином реакция (5) протекает практически мгновенно, со скоростями не позволяющими измерить их обычными кинетическими методами. Из полученных данных следует, что по мере увеличения ковалентности и прочности внутрициклических $\mathrm{NH}-$ связей в ряду $\mathrm{H}_{2} \mathrm{PAR}_{4}$ $\rightarrow \mathrm{H}_{2} \mathrm{Pc}\left(\mathrm{NO}_{2}\right)_{4}\left({ }^{\mathrm{t}} \mathrm{Bu}\right)_{4} \rightarrow \mathrm{H}_{2} \mathrm{Pc}\left({ }^{\mathrm{t}} \mathrm{Bu}\right)_{4}$ протоно-донорная способность молекулы уменьшается.

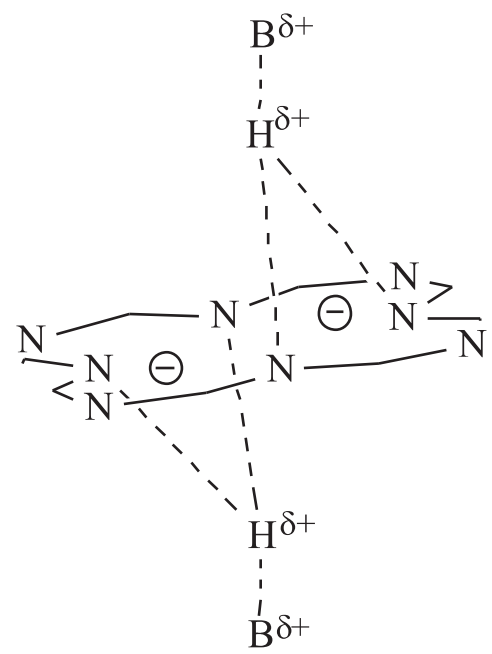

(I)
Таблица 3. Кинетические параметры реакции кислотноосновного взаимодействия $\mathrm{H}_{2} \mathrm{Pc}\left(\mathrm{Bu}^{\mathrm{t}}\right)_{4}$ с основаниями в диметилсульфоксиде $\left(\mathrm{C}_{\mathrm{H}_{2} \mathrm{Pc}\left(\mathrm{Bu}_{4}^{\mathrm{t}}\right)_{4}}^{\mathrm{O}}=0,16 \cdot 10^{-5}\right.$ моль/ $\left./ \mathrm{J}^{[17]}\right)$.

\begin{tabular}{lcc}
\hline Основание & $k^{298}{ }^{2 \phi} \cdot 10^{4}, \mathrm{c}^{-1}$ & $\begin{array}{c}E_{\mathrm{a}}, \\
\text { кДж/моль }\end{array}$ \\
\hline Морфолин & 0,05 & 65 \\
Бензиламин & 0,15 & 52 \\
\hline
\end{tabular}

Согласно [7,16,17], в комплексах $\mathrm{H}_{2} \mathrm{PAR}_{4} \cdot 2 B$, $\left.\mathrm{H}_{2} \mathrm{Pc}\left(\mathrm{NO}_{2}\right)_{4}{ }^{(} \mathrm{Bu}\right)_{4} \cdot 2 B$ и $\mathrm{H}_{2} \mathrm{Pc}\left({ }^{\mathrm{t}} \mathrm{Bu}\right)_{4} \cdot 2 B$ внутрициклические атомы водорода, связанные с молекулами $B$, по-видимому, располагаются над и под плоскостью макроцикла, что обеспечивает соблюдение высокой симметрии распределения зарядов. При этом в малополярном бензоле, обладающим слабовыраженной сольватирующей способностью, полная передача протонов NH-групп от кислоты к основанию, приводящая к возникновению разделенных растворителем ионных пар с последующей их диссоциацией, не наблюдается. Вполне вероятно, что кислотно-основное взаимодействие ограничивается либо стадией образования Н-комплекса (I), либо ионного комплекса, представляющего собой Н-связанную ионную пару (II). ${ }^{[18]}$

Следует, однако, отметить, что вопрос о строении комплексов с переносом протонов является достаточно сложным и требует дальнейшего углубленного изучения.

\section{Выводы}

Показано, что порфиразины в присутствии азотсодержащих оснований проявляют свойства двухосновных NH-кислот и образуют комплексы c переносом протонов, обладающие различной кинетической устойчивостью. Установлено, что кислотно-основное взаимодействие порфиразинов с азотсодержащими основаниями является кинетически контролируемым процессом, характеризующимся необычно низкими скоростями. Выявлена сильная зависимость скорости и активационных параметров

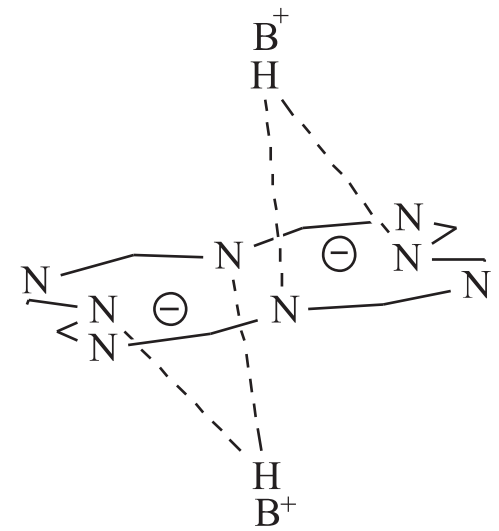

(II) 
кислотно-основного взаимодействия от силы и стерических возможностей основания, а также от кислотных свойств порфиразина.

\section{Список литературы}

\section{References}

1. Stuzhin P.A., Khelevina O.G., Berezin B.D. Azaporphyrins: Acid-Base Properties, in Phthalocyanines: Properties and Applications, Vol. 4 (Leznoff C.C., Lever A.B.P., Eds.) New York: VCH Publishers, 1996, 19 - 77.

2. Stuzhin P.A. J. Porphyrins Phthalocyanines 2003, 7, 813 832.

3. Petrov O.A., Berezin B.D. Zh. Fiz. Khim. 1999, 73, 830 - 834 (in Russ).

4. Petrov O.A., Chizhova N.V. Koord. Khim. 1999, 25, 393 400 (in Russ).

5. Petrov O.A. Zh. Fiz. Khim. 2000, 74, 838 - 842 (in Russ).

6. Petrov O.A. Koord. Khim. 2003, 29, 144 - 150 (in Russ).

7. Petrov O.A. Koord. Khim. 2001, 27, 483 - 492 (in Russ).

8. Berezin B.D., Berezin M.B. Zh. Fiz. Khim. 1989, 63, 3166 3181 (in Russ).
9. Lomova T.N., Berezin D.B. Makrotsiklicheskii Effekt Metalloporfirinov [Metalloporphyrin Macrocyclic Effect], In: Biologicheski Aktivnye Soedineniya $v$ Rastvorakh [Biologically Active Compounds in Solutions] (Kutepov A.M., Ed.) Moskva: Nauka, 2001, 326-362 (in Russ).

10. Schaffer A.M., Gouterman M, Davidson E.R. Theoret. Chim. Acta 1973, 30, 9 - 30.

11. Bureyko S.F., Oktyabr'skii V.P. Kinetika $i$ Kataliz 1985, 26, 804 - 808 (in Russ).

12. Bureyko S.F., Octyabr'skii V.P. Khim. Fizika 1985, 4, 1403 1408 (in Russ).

13. Blackburne I.D., Katritzky A.K., Takeuchi Y. Accounts Chem. Res. 1975, 8, $300-306$.

14. Alibert A., Serzhent E. Konstanty Ionizatsii Kislot i Osnovanii [Ionization Constants of Acids and Bases] Moskva: Khimiya, 1964, 179 p. (in Russ).

15. Berezin B.D. Coordination Compounds of Porphyrins and Phthalocyanines Toronto: Wiley \& Sons, 1978, 280 p.

16. Petrov O.A., Glazunov A.V. Zh. Fiz. Khim. 2006, 80, 1597 1602 (in Russ).

17. Petrov O.A., Osipova G.V., Semeykin A.S., Berezin B.D. Koord. Khim. 2005, 31, 941 - 945 (in Russ).

18. The Molecular Interactions (Rataychik G., Orvill-Thomas U., Eds.) Moskva: Mir, 1984, 599 p. (in Russ). 